\title{
Identification of strut and assembly errors of a 3-PRS serial-parallel machine tool
}

\author{
Hai Wang ${ }^{\text {a }}$, Kuang-Chao Fan ${ }^{\text {b,* }}$ \\ a Department of Mechanical Engineering, Ming-Chi Institute of Technology, Taiwan, ROC \\ ${ }^{\mathrm{b}}$ Department of Mechanical Engineering, National Taiwan University, 1, Sec. 4, Roosevelt Road, Taipei, Taiwan, ROC
}

Received 4 November 2003; received in revised form 1 February 2004; accepted 8 April 2004

\begin{abstract}
In a serial-parallel type machine tool, the parallel spindle platform plays the key role in manipulating three directions of movement. Spatial symmetry of the 3-PRS loops is essential to the machine's systematic accuracy. Currently, however, there is no effective instrument capable of measuring the symmetrical errors of the corresponding joints and strut lengths during structure assembly. In this study, an experimental method is proposed to identify the mechanism symmetric errors of a 3-PRS serial-parallel machine tool during the test run. It is based on the differentiation of the inverse kinematics equations. The mechanism errors could be derived by an identification model. With the aid of a developed 3D laser ball bar to detect the spatial position and orientation of the spindle platform, and three laser Doppler scales to measure three sliders' positions simultaneously, the length errors of three struts and the symmetrical errors of the $R$-joints and $S$-joints can be identified by the optimization technique. This technique can help shop floor engineers to tune the symmetrical errors of the 3-PRS mechanism during machine assembly.
\end{abstract}

(C) 2004 Elsevier Ltd. All rights reserved.

Keywords: 3-PRS platform; Symmetric errors; 3D laser ball bar

\section{Introduction}

It is always a goal to pursue an interesting development in the machine tool industry that holds great promise for improving accuracy and dexterity. Parallel manipulator offers a radically different type of machine structure relative to the traditional serial type machines. It is believed that the inherent mechanical structure of the parallel type machines provides high dexterity, stiffness, accuracy and speed compared to the conventional multi-axis structure $[1,2]$.

In the past, many comprehensive studies and works have been made in the area of parallel manipulators. Most of these articles focused on the discussion of both the analytical and the numerical methods to solve the kinematics of pure-parallel mechanisms. Recently, several papers discuss the accuracy analysis of Stewart platform manipulator or other kinds of parallel manip-

\footnotetext{
${ }^{*}$ Corresponding author. Tel.: +886-2-2362-0032; fax: +886-2-23641186.

E-mail address: fan@ntu.edu.tw (K.-C. Fan).
}

ulators, in which, external sensors were used to measure the end effectors position and orientation, and kinematics model was used to estimate the effect of mechanism errors of the parallel manipulator respect to end effector's positioning accuracy [3-6]. Those kinds of external sensors usually were for a special purpose and complicated to use. These include video sensor imaging a standard grid plate for spatial position [4], laser tracking coordinate measuring system for end effecters spatial position detecting [5], two inclinometers for platform orientation angle detecting [6]. The other way to access the accuracy of parallel manipulator was applying internal sensors which has built in some manipulator joints [7,8]. These sensors were popular and easy to set up, but the final accuracy improvement was limited. There are also some methods employing joint sensors for self-calibration, or autonomous calibration, the kinematic parameters of closeloop chain of the robot or the PKM $[9,10]$.

The parallel type machine tools have not been widely used in industry due to its inadequate accuracy in comparison with conventional serial type machine tools. 
Accuracy enhancement has to be implemented in parts manufacturing and system assembly. In this paper, the symmetric errors of a 3-PRS spindle platform mechanism are identified by experimental method in association with inverse kinematics equations. With the aid of a developed 3D laser ball bar (LBB) to detect the spatial position and orientation of the spindle platform [11], and three laser Doppler scales to measure three sliders' positions simultaneously, the length errors of three struts and the symmetrical errors of the $R$-joints and $S$-joints can be identified by the optimization technique. This technique can help shop floor engineers to tune the symmetrical errors of the 3-PRS mechanism during machine assembly. Some other errors which may also affect the accuracy of the 3-PRS structure are neglected in this study, such as:

1. Tolerance in each joint.

2. Perpendicularity of each column to the base plate.

3. Elastic deformation of each strut.

\section{Machine tool configuration}

The machine tool under investigation is called a serial-parallel type machine tool, as shown in Fig. 1. It consists of a three-degree-of-freedom spindle platform and a conventional two-degree-of-freedom $X-Y$ table to form a 5 -axis structure. The spindle is assembled in the platform, which is connected to three struts of con-

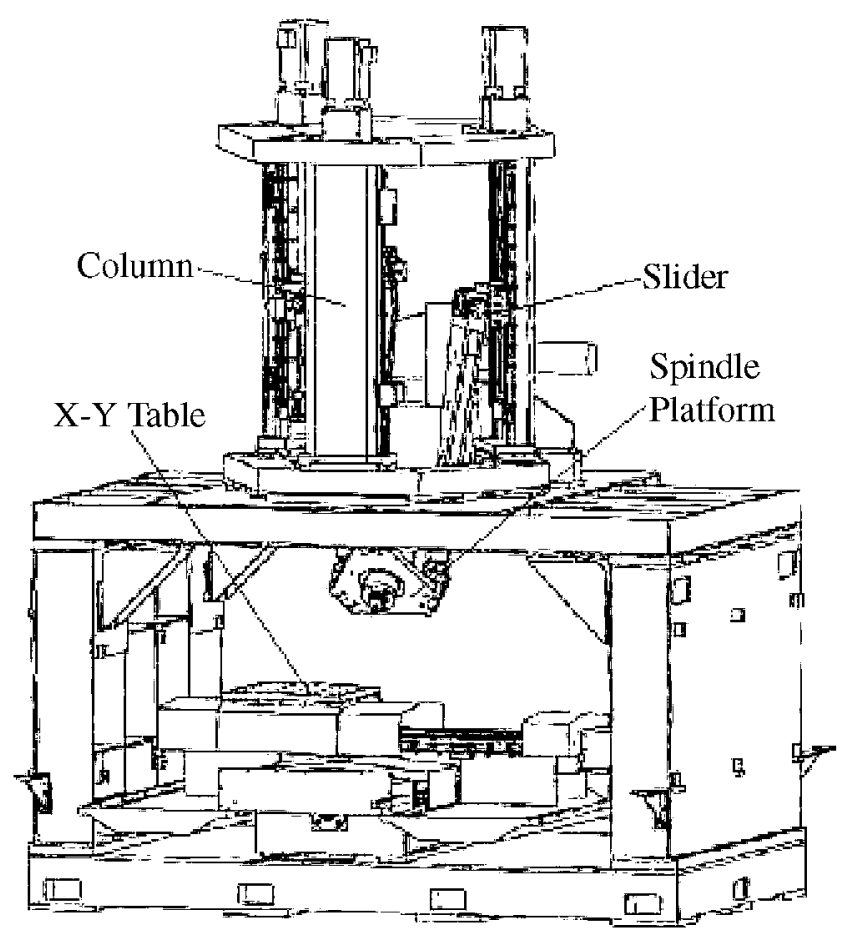

Fig. 1. Structure of the serial-parallel type machine tool. stant length by means of ball joints (or $U$-joint) that are equally spaced at a nominal angle of $120^{\circ}$. The other end of each strut is connected to a slider with a rotational joint. Each slider can move up and down along the corresponding vertical slideway fixed to a column that is also spaced at nominal $120^{\circ}$ angle from one another. The platform, such constructed, has one linear motion in the $Z$-axis and two angular rotations $(\alpha$ and $\beta$ ) in $X$-axis and $Y$-axis, respectively. The $X-Y$ table that supports the workpiece provides the linear motions of the workpiece in two horizontal directions. The features of this configuration are easy to manufacture and control, more accurate and larger workspace, compared to the Stewart platform-based parallel machine tool.

\section{Inverse kinematics analysis}

In order to analyze the kinematics of the parallel mechanism, three relative coordinate frames are assigned, as shown in Fig. 2. A static Cartesian coordinate frame $X Y Z$ is fixed at the base of the machine tool with the $Z$-axis pointing to the vertical direction, the $X$-axis pointing toward $B_{1}$, and the $Y$-axis pointing along the $B_{2} B_{3}$ line. The movable Cartesian coordinate frame, $X^{\prime} Y^{\prime} Z^{\prime}$, is fixed at the center of the $X-Y$ table with the same axes directions as the $X Y Z$ coordinate frame. The third coordinate frame, $x y z$, is assigned to the tool tip, with the $z$-axis coinciding with the spindle axis. The ball joint $b_{1}$ is located in the plane $x_{\mathrm{T}} z . l_{i}$ denotes the $i$ th strut length. $H_{i}$ is the height of $R_{i}$ along the $Z$-axis and $h$ is the distance from the tool tip $o_{\mathrm{T}}$ to the center of the platform. In each closed structural loop of a 3-PRS parallel manipulator, a closed form of vector chain of the linkage can be drawn, as shown in Fig. 3. The related equation can be presented in the following vector form:

$$
\begin{aligned}
{ }^{o} \mathbf{L}_{i} & ={ }^{o} \mathbf{b}_{i}+{ }^{o} \mathbf{p}-{ }^{o} \mathbf{R}_{i}={ }_{{ }^{\mathrm{T}}}{ }^{o}[R] \cdot{ }^{o} \mathbf{b}_{i}+{ }^{o} \mathbf{p}-{ }^{o} \mathbf{R}_{i}, \\
i & =1,2,3
\end{aligned}
$$

where the matrix ${ }_{o_{\mathrm{T}}}^{o}[R]$ describes the relative orientation of the spindle platform coordinate frame $x y z$ to the base coordinate system $X Y Z$, the left upper script $o$ denotes the vector with respect to the base coordinate system $X Y Z$, and the left under script $o_{\mathrm{T}}$ denotes the vector with respect to platform coordinate system $x y z$. $\mathbf{b}_{i}$ is the position vector of the $i$ th ball joints, $\mathbf{p}$ is the position vector of the origin of the frame $x y z$ (tool tip position), $\mathbf{R}_{i}$ is the position vector of the $i$ th $R$-joint, $\mathbf{L}_{i}$ is the $i$ th strut vector.

The inverse kinematics analysis has been derived by the authors [12]. This paper only summarizes some useful equations as below. 

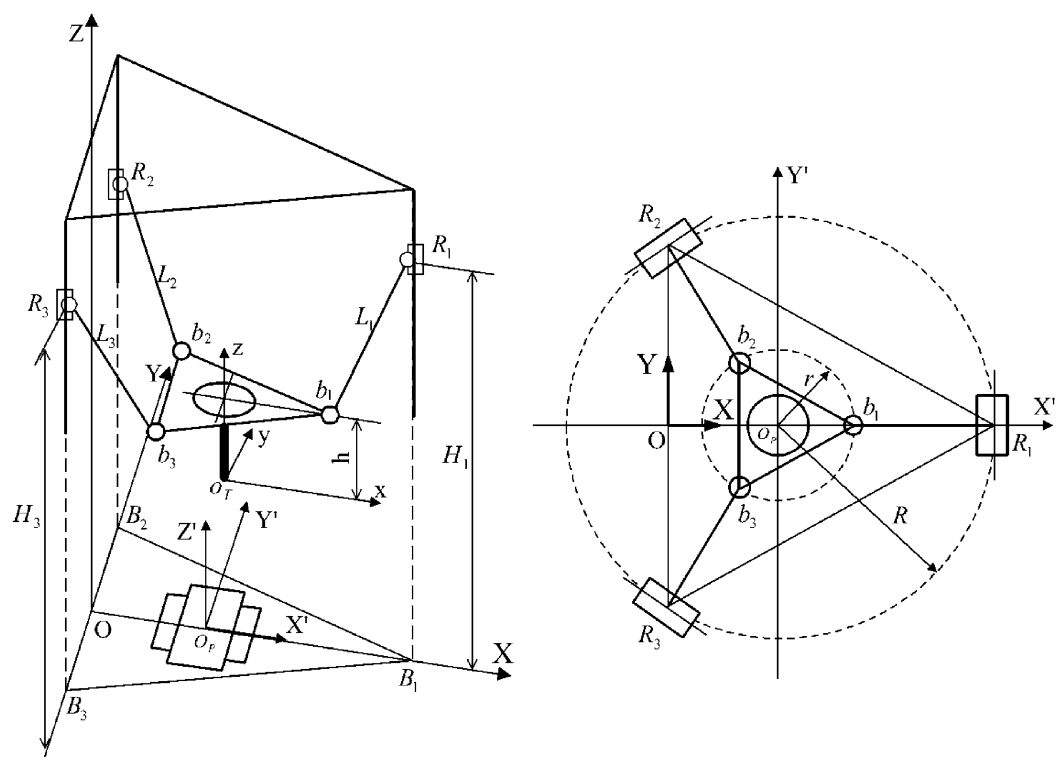

Fig. 2. Schematic diagram of the serial-parallel structure.

Let $R$ and $r$ be the radii of circles passing through joints $R_{i}$ and $b_{i}(i=1-3)$, respectively. The positions of $R_{i}$ referenced to the coordinate frame $X Y Z$ can be expressed by

$$
\begin{aligned}
& \mathbf{R}_{1}=\left[\begin{array}{lll}
\frac{3}{2} R & 0 & H_{1}
\end{array}\right]^{\mathrm{T}} \\
& \mathbf{R}_{2}=\left[\begin{array}{lll}
0 & \frac{\sqrt{3}}{2} R & H_{2}
\end{array}\right]^{\mathrm{T}} \\
& \mathbf{R}_{3}=\left[\begin{array}{lll}
0 & -\frac{\sqrt{3}}{2} R & H_{3}
\end{array}\right]^{\mathrm{T}}
\end{aligned}
$$

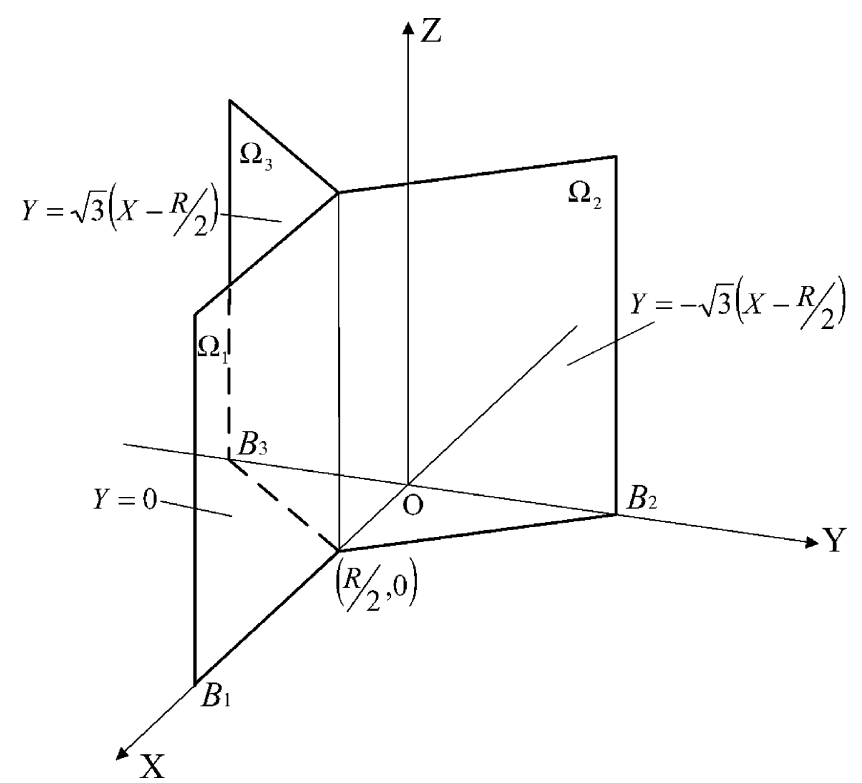

Fig. 3. Illustration of the three constraint planes.
The Cartesian position of the ball joints $\mathbf{b}_{i}$ with respect to the frame $X Y Z$ can be expressed by

$$
\begin{aligned}
& {\left[\begin{array}{l}
\mathbf{b}_{1} \\
1
\end{array}\right]_{o}=\left[\begin{array}{l}
X_{1} \\
Y_{1} \\
Z_{1} \\
1
\end{array}\right]=\left[\begin{array}{l}
r k_{1}+h n_{1}+x_{\mathrm{T}} \\
r k_{2}+h n_{2}+y_{\mathrm{T}} \\
r k_{3}+h n_{3}+z_{\mathrm{T}} \\
1
\end{array}\right]} \\
& {\left[\begin{array}{l}
\mathbf{b}_{2} \\
1
\end{array}\right]_{o}=\left[\begin{array}{l}
X_{2} \\
Y_{2} \\
Z_{2} \\
1
\end{array}\right]=\left[\begin{array}{l}
-\frac{r}{2} k_{1}+\frac{\sqrt{3} r}{2} m_{1}+h n_{1}+x_{\mathrm{T}} \\
-\frac{r}{2} k_{2}+\frac{\sqrt{3} r}{2} m_{2}+h n_{2}+y_{\mathrm{T}} \\
-\frac{r}{2} k_{3}+\frac{\sqrt{3} r}{2} m_{3}+h n_{3}+z_{\mathrm{T}} \\
1
\end{array}\right]} \\
& {\left[\begin{array}{l}
\mathbf{b}_{3} \\
1
\end{array}\right]_{o}=\left[\begin{array}{l}
X_{3} \\
Y_{3} \\
Z_{3} \\
1
\end{array}\right]=\left[\begin{array}{l}
-\frac{r}{2} k_{1}-\frac{\sqrt{3} r}{2} m_{1}+h n_{1}+x_{\mathrm{T}} \\
-\frac{r}{2} k_{2}-\frac{\sqrt{3} r}{2} m_{2}+h n_{2}+y_{\mathrm{T}} \\
-\frac{r}{2} k_{3}-\frac{\sqrt{3} r}{2} m_{3}+h n_{3}+z_{\mathrm{T}} \\
1
\end{array}\right]}
\end{aligned}
$$

where $\left[\begin{array}{lll}x_{\mathrm{T}} & y_{\mathrm{T}} & z_{\mathrm{T}}\end{array}\right]^{\mathrm{T}}={ }^{o} \mathbf{p}$ and the orientation unit vectors $\mathbf{k}=\left[\begin{array}{lll}k_{1} & k_{2} & k_{3}\end{array}\right]^{\mathrm{T}}, \mathbf{m}=\left[\begin{array}{lll}m_{1} & m_{2} & m_{3}\end{array}\right]^{\mathrm{T}}$, and $\mathbf{n}=$ $\left[\begin{array}{lll}n_{1} & n_{2} & n_{3}\end{array}\right]^{\mathrm{T}}$ are the directional cosines of the axes $x, y$ and $z$ with respect to the coordinate frame $X Y Z$. The position component of the rotational joint in the $Z$-axis is

$$
\begin{aligned}
H_{i} & =R_{i Z}=\sqrt{l_{i}^{2}-\left(R_{i X}-b_{i x}\right)^{2}-\left(R_{i Y}-b_{i y}\right)^{2}}+b_{i z}, \\
i & =1,2,3
\end{aligned}
$$

It is called inverse kinematics equations. 
The inverse kinematics problem involves the computation of the position of each of the rotational joint (the slider) through the corresponding ball joint if the spindle platform's position and orientations are known.

\section{Mechanism errors identification model}

Nominally, the large circle passing through three $R$-joints should be concentric with the small circle passing through those three $b$-joints. On the basis of the machine tool configuration, its structure actually consists of three parallel and symmetric structural loops. The positional relation of each structure member in loop is presented in Eq. (1). Expressing strut length in scale formation as

$l_{i}^{2}=\left([R] \cdot \mathbf{b}_{i}+\mathbf{p}-\mathbf{R}_{i}\right)^{\mathrm{T}} \cdot\left([R] \cdot \mathbf{b}_{i}+\mathbf{p}-\mathbf{R}_{i}\right)$

Defining an objective function as

$$
\begin{aligned}
f & =f\left(l_{i}, b_{i x}, b_{i y}, b_{i z}, R_{i X}, R_{i Y}, R_{i Z}\right) \\
& =l_{i}^{2}-\left([R] \cdot b_{i}+p-R_{i}\right)^{\mathrm{T}} \cdot\left([R] \cdot b_{i}+p-R_{i}\right)=0
\end{aligned}
$$

Differentiating Eq. (8), it yields

$$
\begin{aligned}
& 2 l_{i} \mathrm{~d} l_{i}-2\left([R] \cdot \mathbf{b}_{i}+\mathbf{p}-\mathbf{R}_{i}\right)^{\mathrm{T}}[R] \mathrm{d} b_{i} \\
& \quad+2\left([R] \cdot \mathbf{b}_{i}+\mathbf{p}-R_{i}\right)^{\mathrm{T}} \mathrm{d} \mathbf{R}_{i}=0
\end{aligned}
$$

Substituting Eq. (1) into Eq. (9) yields

$$
\begin{aligned}
& \mathbf{L}_{i}^{\mathrm{T}} \mathrm{d} \mathbf{R}_{i}=\left([R] \cdot \mathbf{b}_{i}+\mathbf{p}-\mathbf{R}_{i}\right)^{\mathrm{T}}[R] \mathrm{d} \mathbf{b}_{i}-l_{i} \mathrm{~d} l_{i} \\
& L_{i X} \mathrm{~d} R_{i X}+L_{i Y} \mathrm{~d} R_{i Y}+L_{i Z} \mathrm{~d} R_{i Z} \\
& \quad=\left([R] \cdot \mathbf{b}_{i}+\mathbf{p}-\mathbf{R}_{i}\right)^{\mathrm{T}}[R] \mathrm{d} \mathbf{b}_{i}-l_{i} \mathrm{~d} l_{i}
\end{aligned}
$$

where $\mathrm{d} R_{i z}=\mathrm{d} H_{i}$ denotes the position error of rotation joint in $Z$ direction. Rearrange it to

$$
\begin{aligned}
L_{i Z} \mathrm{~d} H_{i}= & \left([R] \cdot \mathbf{b}_{i}+\mathbf{p}-\mathbf{R}_{i}\right)^{\mathrm{T}}[R] \mathrm{d} b_{i}-L_{i X} \mathrm{~d} R_{i X} \\
& -L_{i Y} \mathrm{~d} R_{i Y}-l_{i} \mathrm{~d} l_{i}
\end{aligned}
$$

Expressing Eq. (10) in a matrix form, it yields

$$
\begin{aligned}
\mathrm{d} H_{i}= & \frac{1}{L_{i Z}}\left[\left([R] \mathbf{b}_{i}+\mathbf{p}-\mathbf{R}_{i}\right)^{\mathrm{T}}[R]\right. \\
& \cdot\left[\begin{array}{l}
\mathrm{d} \mathbf{b}_{i} \\
\mathrm{~d} R_{i X} \\
\mathrm{~d} R_{i Y} \\
\mathrm{~d} l_{i}
\end{array}\right]
\end{aligned}
$$

for $i=1,2,3 . \mathrm{d} H_{i}$ is the deviation of the actual slider position from its ideal position of loop $i$. The ideal slider position $\left(H_{i}^{c}\right)$ can be calculated from the inverse kinematics Eq. (6), the actual slider position $\left(H_{i}^{m}\right)$ has to be measured by instrument.
Substituting Eq. (6) into Eq. (11), it yields

$$
\begin{aligned}
\mathrm{d} H_{i, j}= & H_{i, j}^{m}-H_{i, j}^{c} \\
= & H_{i, j}^{m}-\left(\sqrt{l_{i}^{2}-\left(R_{i X}-b_{i X, j}\right)^{2}-\left(R_{i Y}-b_{i Y, j}\right)^{2}}+b_{i Z, j}\right) \\
= & \frac{1}{L_{i Z, j}}\left[\left([R]_{j} \mathbf{b}_{i}+\mathbf{p}_{j}-\mathbf{R}_{i, j}\right)^{\mathrm{T}}[R]_{j}-L_{i X, j}-L_{i Y, j}-l_{i}\right] \\
& \cdot\left[\begin{array}{l}
\mathrm{d} \mathbf{b}_{i} \\
\mathrm{~d} R_{i X} \\
\mathrm{~d} R_{i Y} \\
\mathrm{~d} l_{i}
\end{array}\right]
\end{aligned}
$$

for $i=1,2,3 ; j=1,2, \ldots, m$, where $i$ denotes the $i$ th structure branch and $j$ indicates the $j$ th measured platform position for total $m$ times. The $j$ th measured spindle platform position can be expressed in a vector form as $\prod_{j}=\left[x_{T j}, y_{T j}, z_{T j}, \alpha_{j}, \beta_{j}, \gamma_{j}\right]^{\mathrm{T}}$, which could be measured by the developed 3D LBB as described in the next section. The overall mechanism error vector $\left(\mathrm{d} U_{i}\right)$ of the $i$ th parallel manipulator investigated here is expressed as

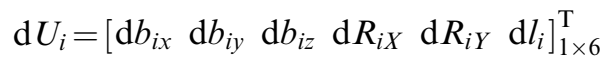

The whole mechanism errors of three parallel manipulators have 18 items. Eq. (12) can be simplified to the following matrix form:

$$
\left[\begin{array}{l}
\mathrm{d} H_{1, j} \\
\mathrm{~d} H_{2, j} \\
\mathrm{~d} H_{3, j}
\end{array}\right]_{3 j \times 1}=\left[\begin{array}{lll}
J_{1} & & \\
& J_{2} & \\
& & J_{3}
\end{array}\right]_{3 j \times 18} \cdot\left[\begin{array}{l}
\mathrm{d} U_{1} \\
\mathrm{~d} U_{2} \\
\mathrm{~d} U_{3}
\end{array}\right]_{18 \times 1}
$$

\section{Measurement principles}

From Eq. (14) it is understood that the 18 terms of mechanism errors can be computed if the spindle's spatial vector $\Pi_{\mathrm{j}}$ and the slider's height errors $\mathrm{d} H_{i, j}$ can be measured at 18 different programmed positions.

\subsection{Measuring equipments}

In order to measure the spindle's spatial vector a $3 \mathrm{D}$ LBB was specially designed, as shown in Fig. 4. The 3D LBB is based on the spherical coordinate principle containing one precision laser linear measurement device and two precision laser rotary encoders in the gimbals base with an extendable ball bar. The movement of the $3 \mathrm{D}$ LBB is generated by the precision end ball, which can be dragged by a magnet socket carried by any moving object. The radial motion $(R)$ of the ball is detected by a laser Doppler scale (LDS, model $109 \mathrm{~N}$, made by Optodyne Co.) whose beam passes through telescope tubes and reflected back by a reflec- 


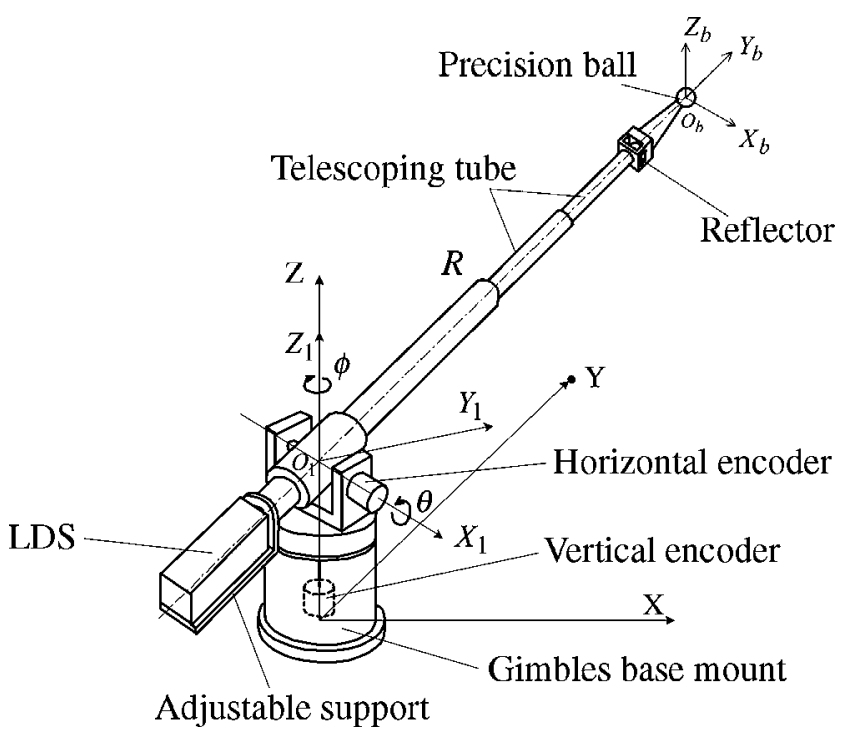

Fig. 4. The structure of 3D LBB.

tor at the bar end. The pitch $(\theta)$ and the yaw $(\phi)$ motions of the bar with respect to the gimbals base are detected by two precision laser rotary encoders (model $\mathrm{K}-1$, made by Canon Co.) individually. Three sensors simultaneously record the ball positions and transform into the Cartesian coordinate in real time. The system possesses the following specifications: $R: 500 \mathrm{~mm}$ range, accuracy $\pm 0.3 \mu \mathrm{m} ; \phi: 35^{\circ}$ range, accuracy \pm 1.15 arc-sec; $\theta: 25^{\circ}$ range, accuracy \pm 1.15 arc-sec [11]. Fig. 5 shows the picture of measuring the spatial position and orientation of the spindle tip with the 3D LBB.

The spatial position of the spindle tip can be directly measured by readings of $(R, \theta, \phi)$ from the $3 \mathrm{D}$ LBB and convert to $o_{\mathrm{T}}\left(x_{\mathrm{T}}, y_{\mathrm{T}}, z_{\mathrm{T}}\right)$. The orientations $(\alpha, \beta)$ have to be obtained by measuring the spindle's vector with the following special strategy. A standard bar was initially inserted in the spindle's tool holder. Its extend-

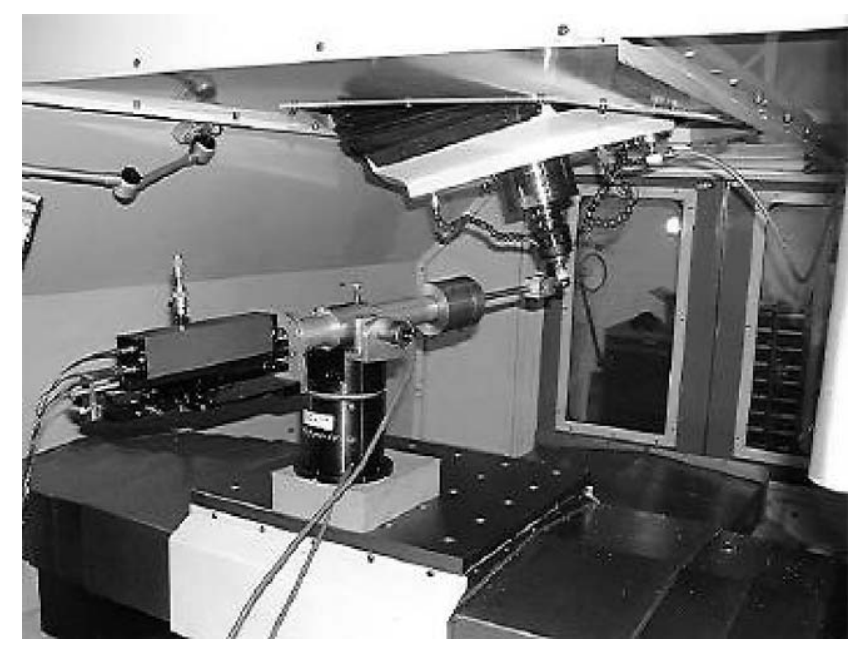

Fig. 5. Measuring the spindle with 3D LBB.

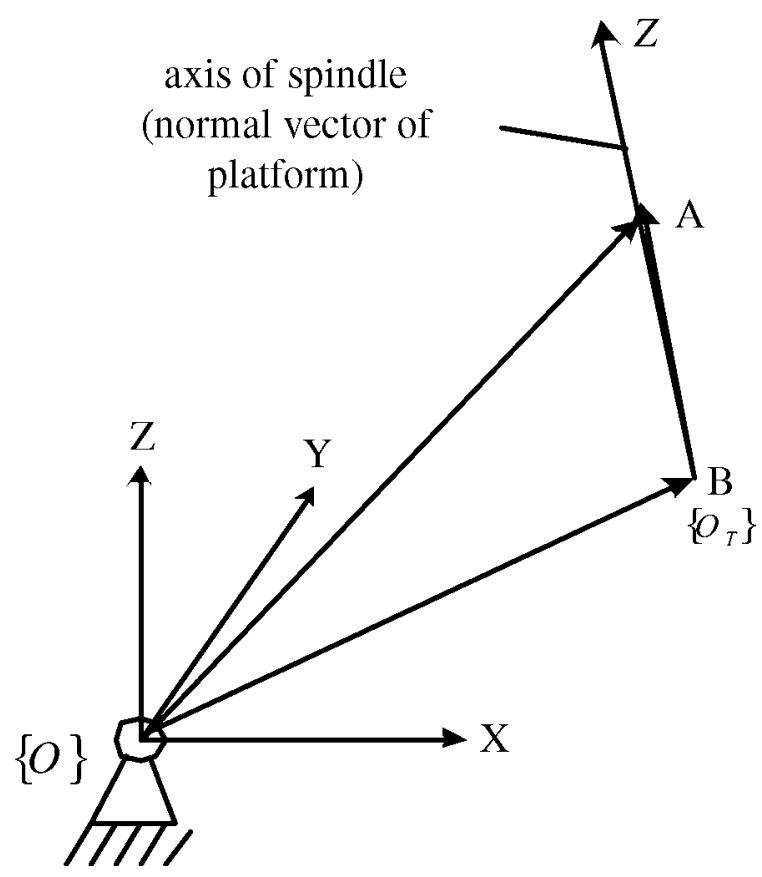

Fig. 6. Spindle orientation measurement.

ing length can be adjusted by a setting screw. This standard bar is a Cr hardened and then ground cylinder, which is purchased from a metrological company. Its cylindricity error is guaranteed by its grade. Usually, the orientation error of the extended standard bar to the center line of the tool holder is very limited if the diameter is fitted. Furthermore, such a limited orientation error is constant in all different spindle poses (orientations). If necessary, we can calibrate this error and compensate it in each reading. This bar is,

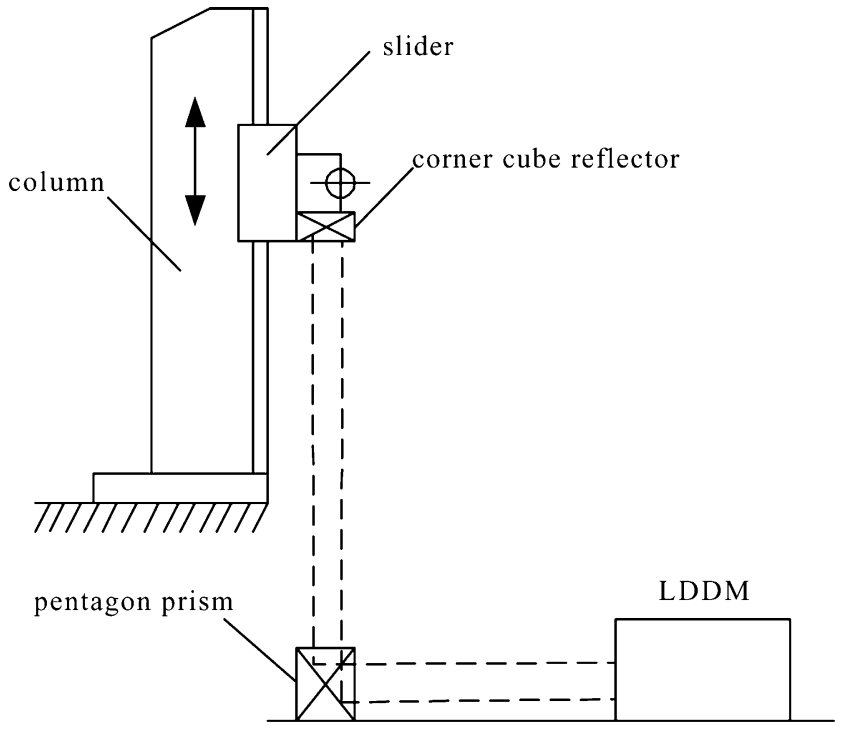

$\mathrm{X}-\mathrm{Y}$ table

Fig. 7. Setup for the measurement of rotational joint movement. 
therefore, assumed to be in line with the normal vector of the spindle platform. At each position the standard bar is initially in its fully retracted length. The first reading of $o_{\mathrm{T}}$ denotes points A, as shown in Fig. 6. The bar is then extended manually to its full length position $\mathrm{B}$ to allow the second reading to be taken. The unit normal vector of spindle platform can be derived by

$$
\begin{aligned}
\overrightarrow{n_{j}} & =\frac{\overrightarrow{A B}}{|\overrightarrow{A B}|}=\left\{n_{1}, n_{2}, n_{3}\right\}^{\mathrm{T}} \\
& =\{\sin \beta,-\sin \alpha \cos \beta, \cos \alpha \cos \beta\}^{\mathrm{T}}
\end{aligned}
$$

The vertical movements of three rotational joints $\left(H_{1}\right.$, $H_{2}, H_{3}$ ) can be simultaneously measured by three laser Doppler scales (LDS) of the same type as in the 3D LBB. The setup is shown in Fig. 7.

\subsection{Measuring procedures}

Measuring procedures are summarized as follows:

(a) The $3 \mathrm{D}$ LBB is precisely aligned so that its extending bar is coincident with the $Y$-axis of the investigated serial-parallel machine firstly. The machine spindle then carries the end ball of 3D LBB with a magnetic socket moving along the $Y$-axis. The two angular encoders should have no readout changes in the pitch and the yaw directions.

(b) Program the spindle to move around in the working space for at least 18 different positions.

(c) At each position $j$, point $A_{j}$, take readings of $(R, \theta, \phi)$ from the $3 \mathrm{D} \mathrm{LBB}$ and convert to $o_{\mathrm{T}}\left(x_{\mathrm{T}}, y_{\mathrm{T}}, z_{\mathrm{T}}\right)$. In the meantime, take readings of the three positions of rotational joints $\left(H_{1 j}, H_{2 j}\right.$, $H_{3 j}$ ) by three LDS simultaneously.

(d) Extend the standard bar to point $B_{j}$, take the second readings of the $3 \mathrm{D} \mathrm{LBB}$, and compute the corresponding spindle orientation $(\alpha, \beta)$ with Eq. (15). It is noted from this equation that the angle $\gamma$ is a function of $\alpha$ and $\beta$.

(e) Repeat steps (b)-(d) until all data sets are collected.

\section{Error identification procedures and experimental results}

From Section 3 it is noted that, if the nominal strut lengths $\left(l_{1}, l_{2}, l_{3}\right)$ and the nominal $R$-joint radius $R$ are given, and if the spindle's position and orientation are measured, the nominal $R$-joint positions $\left(R_{i X}, R_{i Y}\right.$, for $i=1,2,3$ ) can be obtained from Eq. (2), the nominal ball joint positions $\left(\mathbf{b}_{1}, \mathbf{b}_{2}, \mathbf{b}_{3}\right)$ can be computed from Eq. (3)-(5), and then the theoretical rotational joint position can be computed by Eq. (6). Due to the manufacturing errors of the strut length and the assembly errors of the ball joints and $R$-joints, the actual mea- sured $R$-joint vertical movement is different from for each loop. It is based on the earlier assumptions that if the effect of elastic deformation and clearance between joint members are neglected. This is called the inverse kinematics error as expressed by

$F_{i j}=H_{i j}^{m}-H_{i j}^{c}, \quad i=1,2,3 ; \quad j=1,2, \ldots, m$

In other words, if the actual mechanism parameters $b_{i x}, b_{i y}, b_{i z}, R_{i X}, R_{i Y}$ and $l_{i}(i=1,2,3)$ can be identified, $H_{i}^{c}$ and $H_{i}^{m}$ must be the same for any spatial position $j$. A simplified optimization technique by iteration method is proposed to find these actual design parameters.

Defining an objective function as

$C_{i j}=\sum_{j=1}^{m} \sum_{i=1}^{3} F_{i j}, \quad i=1,2,3 ; \quad j=1,2, \ldots, m$

where $C_{i j}$ is a function of design parameters $\left(b_{i x}, b_{i y}\right.$, $b_{i z}, R_{i X}, R_{i Y}, l_{i}$ for $\left.i=1,2,3\right)$. The optimization process is to search the optimum design parameters so that the objective function is minimized. A criterion value ( $\varepsilon=0.01 \mathrm{~mm})$ is set for judging the iteration convergence. Following procedures are employed for the iterative regression algorithm.

(a) Assign the designed nominal values of $b_{i x}, b_{i y}$, $b_{i z}, R_{i X}, R_{i Y}$ and $l_{i}(i=1,2,3)$ as the initial values of the design parameters in optimization process. These initial nominal values are listed in Table 1 . Set the iteration number $k=1$.

(b) Calculate the theoretical rotation joint movement $H_{i j}^{c}(i=1,2,3 ; j=1,2, \ldots, m)$ by the inverse kinematics Eq. (6).

(c) Calculate the inverse kinematics error $F_{i j}=$ $H_{i j}^{m}-H_{i j}^{c}(i=1,2,3 ; j=1,2, \ldots, m)$.

Table 1

The nominal mechanism parameters, unit: $\mathrm{mm}$

\begin{tabular}{lcclcrl}
\hline Branch & $\boldsymbol{b}_{i x}$ & \multicolumn{1}{c}{$\boldsymbol{b}_{i y}$} & $\boldsymbol{b}_{i z}$ & $\boldsymbol{R}_{i X}$ & \multicolumn{1}{c}{$\boldsymbol{R}_{i Y}$} & $\boldsymbol{l}_{i}$ \\
\hline 1 & 200 & 0.00 & 340.722 & 349 & 0.00 & 1107 \\
2 & -100 & 173.205 & 340.722 & -174.5 & 302.243 & 1107 \\
3 & -100 & -173.205 & 340.722 & -174.5 & -302.243 & 1107 \\
\hline
\end{tabular}

Table 2

Twenty-two spindle poses selected for experimental measurement

\begin{tabular}{lrrrrrrrrrrr}
\hline $\begin{array}{l}\text { ID. } \\
\text { no. }\end{array}$ & 1 & 2 & 3 & 4 & 5 & 6 & 7 & 8 & 9 & 10 & 11 \\
\hline$\alpha$ & $0^{\circ}$ & $0^{\circ}$ & $0^{\circ}$ & $0^{\circ}$ & $0^{\circ}$ & $0^{\circ}$ & $0^{\circ}$ & $10^{\circ}$ & $20^{\circ}$ & $35^{\circ}$ & $-10^{\circ}$ \\
$\beta$ & $0^{\circ}$ & $10^{\circ}$ & $20^{\circ}$ & $35^{\circ}$ & $-10^{\circ}$ & $-20^{\circ}$ & $-35^{\circ}$ & $0^{\circ}$ & $0^{\circ}$ & $0^{\circ}$ & $0^{\circ}$ \\
ID. & 12 & 13 & 14 & 15 & 16 & 17 & 18 & 19 & 20 & 21 & 22 \\
no. & & & & & & & & & & \\
\hline$\alpha$ & $-20^{\circ}$ & $-35^{\circ}$ & $5^{\circ}$ & $10^{\circ}$ & $-5^{\circ}$ & $-10^{\circ}$ & $-5^{\circ}$ & $-10^{\circ}$ & $5^{\circ}$ & $10^{\circ}$ & $25^{\circ}$ \\
$\beta$ & $0^{\circ}$ & $0^{\circ}$ & $10^{\circ}$ & $25^{\circ}$ & $-10^{\circ}$ & $-25^{\circ}$ & $10^{\circ}$ & $25^{\circ}$ & $-10^{\circ}$ & $-25^{\circ}$ & $10^{\circ}$ \\
\hline
\end{tabular}


Table 3

Identified mechanism parameters, unit: $\mathrm{mm}$

\begin{tabular}{lrrrrrr}
\hline Branch & \multicolumn{1}{l}{$\boldsymbol{b}_{i x}$} & \multicolumn{1}{c}{$\boldsymbol{b}_{i y}$} & \multicolumn{1}{c}{$\boldsymbol{b}_{i z}$} & \multicolumn{1}{c}{$\boldsymbol{R}_{i X}$} & \multicolumn{1}{c}{$\boldsymbol{R}_{i Y}$} & \multicolumn{1}{l}{$\boldsymbol{l}_{i}$} \\
\hline 1 & 199.96 & 0.012 & 341.100 & 350.587 & 1.186 & 1107.59 \\
2 & -99.98 & 173.21 & 340.630 & -173.721 & 303.594 & 1107.66 \\
3 & -99.95 & -173.22 & 340.890 & -173.238 & -302.730 & 1107.52 \\
\hline
\end{tabular}

Table 4

Identified mechanism errors, unit: $\mathrm{mm}$

\begin{tabular}{lrrrrrl}
\hline Branch & \multicolumn{1}{c}{$\Delta \boldsymbol{b}_{i x}$} & \multicolumn{1}{c}{$\Delta \boldsymbol{b}_{i y}$} & \multicolumn{1}{c}{$\Delta \boldsymbol{b}_{i z}$} & $\Delta \boldsymbol{R}_{i X}$ & \multicolumn{1}{c}{$\Delta \boldsymbol{R}_{i Y}$} & $\Delta \boldsymbol{l}_{i}$ \\
\hline 1 & -0.034 & 0.012 & 0.378 & 1.587 & 1.186 & 0.592 \\
2 & 0.019 & 0.011 & -0.092 & 0.779 & 1.351 & 0.664 \\
3 & 0.043 & -0.021 & 0.168 & 1.262 & -0.487 & 0.526 \\
\hline
\end{tabular}

(d) Compare the objective function $C_{i j}$ to $\varepsilon$. If $C_{i j}$ is less than or equal to $\varepsilon$, the search is terminated. If not, compute the mechanism errors with Eq. (14) $\left(\mathrm{d} U_{k}=J_{k}^{-1} \mathrm{~d} H_{k}\right)$.

(e) Replace the design parameters by $\left(U_{k+1}=\right.$ $U_{k}+\mathrm{d} U_{k}$ ), let $k=k+1$, and go to step (b).

In the experiment, 22 sets of spindle platform poses were programmed in the working volume. These were uniformly selected from the rotational ranges of $\alpha$ and $\beta$ angles, as listed in Table 2. Its corresponding spatial positions and orientations were recorded by a 3D LBB. In the meantime, three independent sets of LDS detected the actual position of each rotation joint. After the iteration procedures, the final actual mechanism parameters were computed as listed in Table 3. The relative mechanism errors are listed in Table 4. Comparing to

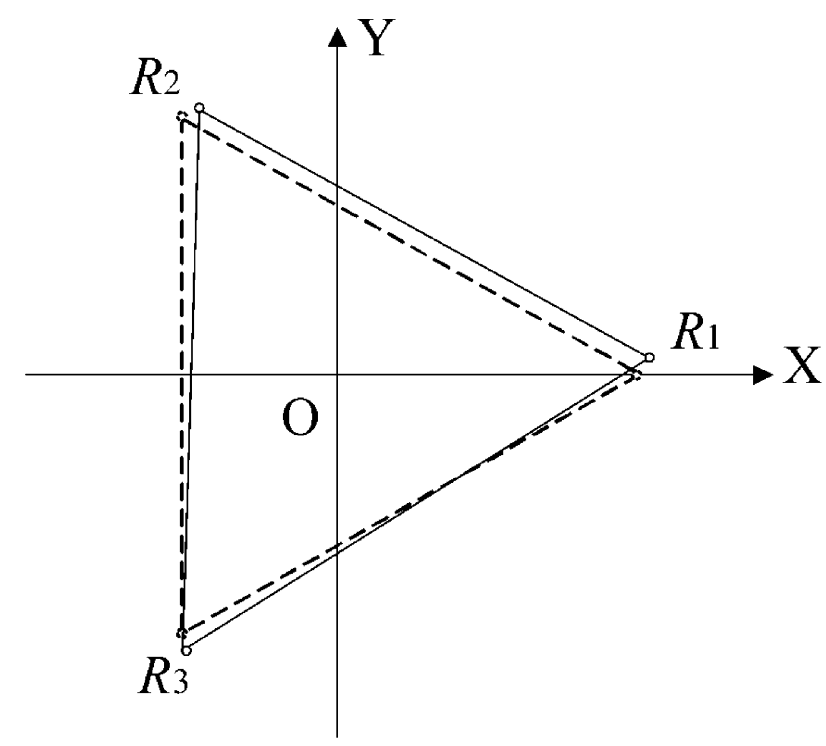

\section{o- - o nominal position $\longleftarrow$ identified position}

Fig. 8. The diagrammatic position error of rotation joints.

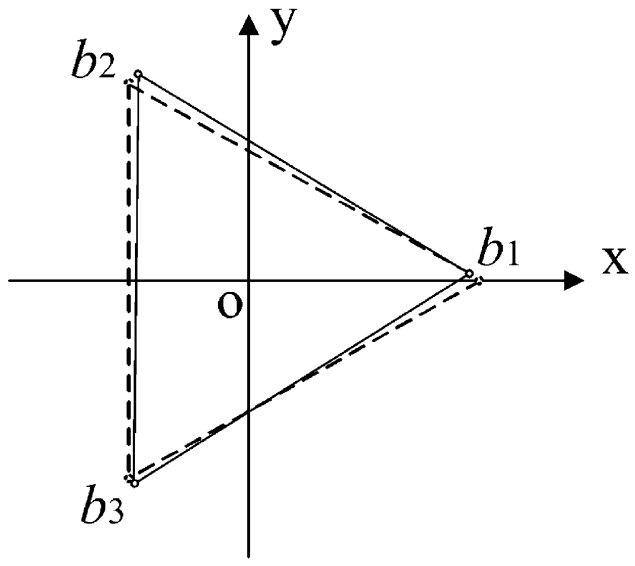

$\sim--\circ$ nominal position $\curvearrowleft \longrightarrow$ identified position

Fig. 9. The diagrammatic position error of ball joints.

the calculated 18 error terms of this study, the measuring noise of the 3D LBB is very small [11]. The noise effect was, therefore, ignored in this study. The geometrical position errors of rotation joints and ball joints of planar view are illustrated in Figs. 8 and 9, respectively. It can be seen that to this investigated machine the assembly error of the $R$-joint is a critical factor to the machine accuracy. It could be caused by the difficulty in precise assembly of three parallel columns to perfect symmetry. The length of each strut was also longer than the design value. It has to be noted that, due to direct connection, the position error of $R$-joint will affect the position of $b$-joint. This machine tool is equipped with Siemens controller. Although individual strut length can be programmed to its correct dimension, the joint position errors have to be corrected by manual reassembly of column positions. These findings were recognized and accepted by the manufacturer for further rework.

\section{Conclusions}

In this paper, a new mechanism errors identification method is proposed for a 3-PRS serial-parallel machine tool. It is based on the inverse kinematics model and volumetric error measurement techniques by the developed 3D LBB assisted by a standard bar. Experimental results in association with the optimization technique can realize the exact mechanism errors of the investigated machine, namely the structural symmetricity errors of the struts and joint locations. This method can help the machine tool builder to adjust the built-in parameters (strut lengths) in NC programming and make fine tuning (joint or column positions) in the assembly stage. 


\section{References}

[1] J. Wang, O. Masory, On the accuracy of a Stewart platformPart I: the effect of manufacturing tolerances, Proceedings of the IEEE International Conference on Robotics and Automation, Atlanta, USA, 2-6 May, 1993, pp. 114-120.

[2] O. Masory, J. Wang, H. Zhuang, On the accuracy of a Stewart platform-Part II: kinematic calibration and compensation, Proceedings of the IEEE International Conference on Robotics and Automation, Atlanta, USA, 2-6 May, 1993, pp. 725-731.

[3] M.P. Olivers, Global kinematic calibration of a Stewart platform, Transaction of the ASME Dynamic System and Control 2 (1995) 57-61.

[4] P. Maurine, E. Dombre, A calibration procedure for the parallel robot delta 4, Proceedings of the IEEE International Conference on Robotics and Automation, Minneapolis, Minnesota, USA, April 22-28, 1996, pp. 975-980.

[5] Y. Koseki, Design and accuracy evaluation of high-speed and high precision parallel mechanism, Proceedings of the IEEE International Conference on Robotics and Automation, Leuven, Belgium, May 16-21, 1998, pp. 1340-1345.

[6] S. Besnard, W. Khalil, Calibration of parallel robots using two inclinometers, Proceedings of the IEEE International Conference on Robotics and Automation, Michigan, USA, 10-15 May, 1999, pp. $1758-1763$.

[7] C.W. Wampler, An implicit loop method for kinematics calibration and its application to closed-chain mechanisms, Proceedings of the IEEE International Conference on Robotics and Automation, Nagoya, Japan, 21-27 May, 1995, pp. 358-364.

[8] H. Zhuang, Autonomous calibration of hexapod machine tools, Transactions of the ASME Manufacturing Science and Technology 1 (2000) 140-148.

[9] D.J. Bennett, J.M. Hollerbach, Autonomous calibration of single-loop closed kinematic chains formed by manipulators with passive endpoint constraints, IEEE Transactions on Robotics and Automation 7 (5) (1991) 597-606.

[10] L. Notash, R.P. Podhorodeski, Kinematic calibration of parallel manipulators, Proceedings of IEEE Conference on Systems, Man, and Cybernetics, 1995, pp. 3310-3315.

[11] K.C. Fan, H. Wang, F.J. Shiou, C.W. Ke, Development of a 3D laser ball bar for the volumetric error measurement of multi-axis machine, Transactions of the NAMRC of SME 31 (2003) 249 256.

[12] K.C. Fan, H. Wang, J.W. Zhao, T.H. Chang, Sensitivity analysis of the 3-PRS parallel kinematic platform of a serial-parallel machine tool, International Journal of Machine Tools and Manufacture 43 (15) (2003) 1561-1569. 